\title{
Ecocardiografia de cães da raça Yorkshire Terrier clinicamente normais
}

\author{
[Echocardiography of clinically normal Yorkshire Terrier dogs] \\ M.G. Castro $^{1}$, R.C.S. Tôrres ${ }^{2}$, R.B. Araújo ${ }^{2}$, R.A.L. Muzzi ${ }^{3}$, E.F. Silva ${ }^{1}$ \\ ${ }^{1}$ Aluno de pós-graduação - Escola de Veterinária - UFMG - Belo Horizonte, MG \\ ${ }^{2}$ Departamento de Clínica e Cirurgia Veterinárias - UFMG - Belo Horizonte, MG \\ ${ }^{3}$ Departamento de Medicina Veterinária - UFLA - Lavras, MG
}

\begin{abstract}
RESUMO
Determinaram-se os parâmetros ecocardiográficos em modo-M normais de cães da raça Yorkshire Terrier. Foram utilizados 30 cães clinicamente sadios, com peso médio de $2,42 \pm 0,64 \mathrm{~kg}$ e idades entre um e seis anos. $\mathrm{O}$ diâmetro do átrio esquerdo e da aorta, a dimensão interna do ventrículo esquerdo na diástole e na sístole, a espessura do septo interventricular na diástole e na sístole, e a espessura da parede posterior do ventrículo esquerdo na diástole e na sístole correlacionaram-se com o peso corporal. As demais variáveis do modo-M não se correlacionaram com peso corporal, sexo ou idade. Os valores ecocardiográficos obtidos neste estudo podem ser utilizados como referência para cães dessa raça.
\end{abstract}

Palavras-chave: cão, ecocardiografia, modo-M, coração

\begin{abstract}
The aim of this study was to establish the normal echocardiographic parameters in M-mode for Yorkshire Terrier dogs. Thirty clinically normal dogs with mean weight of $2.42 \pm 0.64 \mathrm{~kg}$ and ages varying from one to six years old were studied. The left atrial diameter, the aortic diameter, the left ventricular internal dimension at end-diastole and end-systole, end-diastolic and end-systolic interventricular septal thickness, and end-diastolic and end-systolic left ventricular posterior wall thickness had correlation with body weight. The other M-mode variables showed no linear correlations with body weight, sex or age. The echocardiographic values found in this study can be used as reference for this breed.
\end{abstract}

Keywords: dog, ecocardiography, M-mode, heart

\section{INTRODUÇÃO}

A ecocardiografia é definida como o exame ultrassonográfico do coração e grandes vasos, e, nas últimas duas décadas, tornou-se um dos mais importantes métodos de diagnóstico em cardiologia veterinária (Boon, 1998; Kienle, 1998; Kienle e Thomas, 2005).

Dentre as principais limitações do uso dos parâmetros e dos índices ecocardiográficos está a variação entre as diferentes raças de cães. Morrison et al. (1992) realizaram um estudo pelo qual foram avaliadas quatro raças caninas com o objetivo de estabelecer os valores de normalidade para as medidas ecocardiográficas, em modo-M, e comparar as variações destas entre as quatro raças estudadas. Os autores concluíram que a raça, considerando o peso corporal, é fator importante que influencia, de forma significativa, as mensurações dos parâmetros ecocardiográficos dos cães. Concluíram ainda que variações destas mensurações podem ocorrer em animais da mesma raça, devido à variação de tamanho e peso corporal.

Outros estudos foram realizados com os objetivos de padronizar os valores ecocardiográficos em modo-M para algumas raças caninas, como Pastor Alemão, Poodle Miniatura, Golden Retriever, Boxer e Schnauzer Miniatura (Muzzi et al., 2000; Yamato et al., 2006; Pellegrino et al., 2007; Silva et al., 2008), e avaliar as alterações ecocardiográficas que podem ser indicativas de cardiopatias ou apenas serem características inerentes à raça estudada (Page et al., 1993; Snyder et al., 1995).

Recebido em 23 de junho de 2010

Aceito em 4 de julho de 2011

E-mail: michellegc@ig.com.br 
Animais da raça Yorkshire Terrier são frequentemente acometidos por cardiopatias congênitas, como a persistência do ducto arterioso, e por afecções adquiridas, como a endocardiose da valva mitral (Castro et al., 2009). Além disso, essa raça é considerada como uma das mais predispostas ao colapso de traqueia (McReady et al., 2007; Ferian, 2009), afecção respiratória crônica que, além de apresentar sinais clínicos em comum com algumas cardiopatias, possibilita o desenvolvimento de importantes alterações no sistema cardiovascular (Marolf et al., 2007).

Pela grande variabilidade racial dos valores ecocardiográficos e pela escassez de estudos visando à avaliação cardíaca de cães hígidos da raça Yorkshire Terrier, o objetivo deste trabalho foi determinar os parâmetros ecocardiográficos em modo-M normais para esta raça.

\section{MATERIAL E MÉTODOS}

Foram utilizados 30 cães da raça Yorkshire Terrier clinicamente normais, sendo 14 machos e 16 fêmeas, com peso inferior a $4 \mathrm{~kg}$ e idades entre um e seis anos, de origem variada. Os cães foram selecionados por meio de exames clínico, eletrocardiográfico e radiográfico do tórax, além de avaliação laboratorial, que incluía a realização de hemograma completo, dosagem sérica de ureia, creatinina, alanina aminotransferase, aspartato aminotransferase, bilirrubinas, colesterol total, fosfatase alcalina, gama glutamil transpeptidase, proteínas totais e albumina. Os considerados hígidos foram submetidos ao exame ecocardiográfico.

Os animais selecionados foram submetidos a uma sedação leve com maleato de acepromazina (Acepram 0,2\%, 0,03mg/kg Univet SA), por via intravenosa. Após 10 minutos de repouso, os cães foram encaminhados ao setor de cardiologia, onde foram posicionados em mesa apropriada em decúbito lateral, por meio de contenção manual. Para realização do exame ecocardiográfico, foi utilizado um aparelho de ultrassonografia (Ecocardiógrafo HP Sonos 100 CF - Hewlett Packard) composto por modos bidimensional, $\mathrm{M}$ e Doppler - pulsado, contínuo e por mapeamento em cores -, com transdutor de varredura setorial mecânica de 2,5 a $7,5 \mathrm{MHz}$ e acompanhamento eletrocardiográfico no monitor, fornecendo a frequência cardíaca. Todos os exames foram gravados em videoteipe (Vídeo Gravador Sony - Sony Corporation) para posterior avaliação. Foram realizadas três medidas de cada variável em diferentes ciclos cardíacos e analisadas as suas médias.

Para a realização do modo bidimensional, adotaram-se as recomendações de Thomas et al. (1993) e Boon (1998). As imagens foram obtidas com os animais em decúbito lateral, utilizando-se as regiões paraesternal direita e esquerda (janelas acústicas). Foram avaliados as câmaras cardíacas, a contratilidade miocárdica e o aspecto morfológico das valvas cardíacas. Na posição paraesternal transversal direita, na região da base cardíaca, foram obtidos os diâmetros do átrio esquerdo (AE) e da aorta (AO), no início da diástole, segundo Rishniw e Erb (2000), para calcular a relação entre esses parâmetros. A AO foi mensurada ao longo da linha de fechamento das cúspides coronariana direita e não coronariana. O AE foi mensurado a partir de uma linha paralela à linha de fechamento das cúspides não coronariana e coronariana esquerda (Fig. 1). As imagens do modo bidimensional serviram de orientação para realização dos demais modos.

No modo-M, foram obtidas, ao final da sístole e da diástole, a dimensão interna do ventrículo esquerdo (VE) e as espessuras do septo interventricular (SIV) e da parede posterior do ventrículo esquerdo (PVE), de acordo com Lombard (1984).

A partir das medidas citadas, foram calculados, segundo Boon et al. (1983), a relação da espessura do septo interventricular e da parede posterior no final da diástole (SIVd:PVEd), a relação da espessura do septo interventricular e da parede posterior no final da sístole (SIVs:PVEs), o percentual de encurtamento sistólico do ventrículo esquerdo $(\% \Delta \mathrm{D})$, o percentual de espessamento sistólico do septo interventricular (\%ESS) e da parede posterior do ventrículo esquerdo (\%ESP) e a velocidade de encurtamento circunferencial da fibra cardíaca (Vcf). O volume sistólico final (VSF) foi calculado pelo método de Teichholz pelo próprio programa do aparelho. O volume diastólico final (VDF), necessário para o cálculo da fração de ejeção (FEj), foi obtido da mesma maneira. Ainda no modo-M, foi mensurada a distância entre o ponto $\mathrm{E}$ do folheto anterior da valva mitral e o septo interventricular (EPSS). 


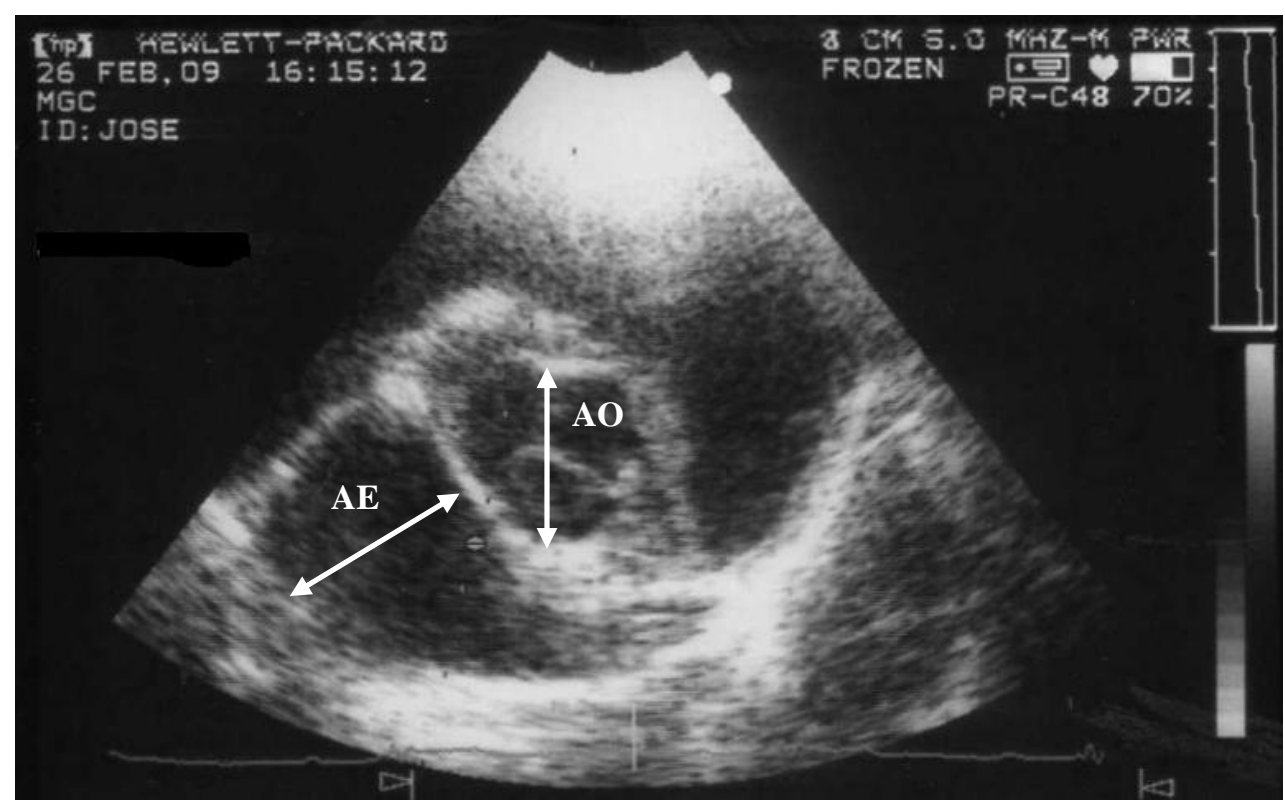

Figura 1. Imagem ecocardiográfica em modo bidimensional de cão da raça Yorkshire Terrier. Posição paraesternal transversal direita, na região da base cardíaca. Observam-se os pontos de mensuração do diâmetro do átrio esquerdo (AE) e da aorta (AO) (setas).

Os valores dos parâmetros ecocardiográficos obtidos neste estudo foram submetidos à estatística descritiva para o cálculo do valor médio e do desvio-padrão. O coeficiente de correlação de Pearson (r) foi calculado com a finalidade de avaliar a correlação entre as variáveis peso corporal e idade e os valores ecocardiográficos mensurados. Considerou-se correlação positiva e significativa quando o coeficiente de correlação foi maior ou igual a 0,40 e a significância $(\mathrm{P})$ menor ou igual a 0,05 . Em relação ao sexo, foi utilizado o teste $\mathrm{t}$ para comparação entre machos e fêmeas (Sampaio, 2002).

\section{RESULTADOS E DISCUSSÃO}

A faixa etária variou de um a seis anos de idade, com média de 2,74 $\pm 1,24$ anos, e o peso variou de 1,5 a $4,0 \mathrm{~kg}$, com média de $2,42 \pm 0,64 \mathrm{~kg}$ Os machos pesaram em média $2,43 \pm 0,67 \mathrm{~kg}$, e as fêmeas $2,42 \pm 0,65 \mathrm{~kg}$. A amostra constituiu-se de $53 \%$ de fêmeas e $47 \%$ de machos.
O protocolo sedativo utilizado neste experimento permitiu a realização do exame ecocardiográfico apenas com discreta contenção manual dos animais. Os cães se apresentaram, em sua maioria, taquipneicos antes da administração do ansiolítico, após o que mostraram padrão respiratório normal, diminuindo os artefatos durante os exames. Sabe-se que a agitação dos animais e o padrão respiratório alterado podem interferir nas mensurações ecocardiográficas (Boon, 1998). Segundo Cavalcanti et al. (2007) e Silva et al. (2008), a frequência cardíaca mensurada antes e após 10 minutos da aplicação de acepromazina apresenta diferença de valores $(\mathrm{P}<0,01)$, o que foi confirmado neste estudo (Tab. 1). Esses autores observaram que o uso da acepromazina a $0,03 \mathrm{mg} / \mathrm{kg}$ reduz a ansiedade dos cães, mas não altera significativamente os índices ecocardiográficos. O comportamento tranquilo dos animais é fundamental para a determinação dos parâmetros ecocardiográficos normais.

Tabela 1. Frequência cardíaca média (bpm) antes e após 10 minutos da aplicação de acepromazina em 30 cães da raça Yorkshire Terrier

\begin{tabular}{lc}
\hline & Média \pm desvio-padrão \\
\hline Antes da aplicação & $119,33 \pm 21,49 \mathrm{a}$ \\
Após 10 minutos da aplicação & $98,43 \pm 19,94 \mathrm{~b}$ \\
\hline
\end{tabular}

Letras diferentes na coluna indicam diferença entre os valores $(\mathrm{P}<0,05)$. 
Imagens ecocardiográficas satisfatórias foram qualitativamente similares às descritas por Lombard (1984), Thomas et al. (1993) e Boon (1998). A média, o desvio-padrão e o coeficiente de variação de todas as variáveis estão sumarizados na Tab. 2.

O diâmetro do átrio esquerdo (AE) e da aorta (AO) assume especial importância, uma vez que sua relação (AE:AO) é um dos índices mais utilizados na avaliação do tamanho atrial esquerdo (Boon et al., 1983; Rishniw e Erb, 2000; Hansson et al., 2002). As mensurações podem ser realizadas no modo $\mathrm{M}$ ou no bidimensional, porém, segundo Rishniw e Erb (2000) e Hansson et al. (2002), o ecocardiograma bidimensional apresenta maior sensibilidade por resultar em medidas mais criteriosas. No modo-M, o diâmetro do AE inclui somente a dimensão da aurícula esquerda, sendo que o corpo principal do átrio não é considerado, subestimando seu tamanho. $\mathrm{O}$ valor médio da relação AE:AO obtido neste trabalho foi de $1,37 \pm 0,12$, resultado semelhante ao encontrado por Rishniw e Erb (2000), que avaliaram cães de várias raças e com pesos entre 4,0 e $56,0 \mathrm{~kg}$ e obtiveram o valor médio de 1,31. Segundo esses autores, a relação AE:AO maior que 1,6 sugere dilatação atrial esquerda, uma vez que a medida da aorta praticamente não se altera no cão. A relação $\mathrm{AE}: \mathrm{AO}$ não apresentou correlação significativa com as variáveis peso corporal, idade e sexo, aumentando sua aplicabilidade clínica. A correlação entre o diâmetro do AE e da AO foi positiva e significativa com o peso corporal $(r=0,71$ e 0,56 , respectivamente; $\mathrm{P}<0,0006)$, e não se correlacionou com a idade ou o sexo dos animais. Os achados confirmam os já observados por Rishniw e Erb (2000), Hansson et al. (2002) e Yamato et al. (2006).

Tabela 2. Médias, desvios-padrão e coeficientes de variação dos parâmetros e índices ecocardiográficos avaliados em cães da raça Yorkshire Terrier

\begin{tabular}{lcc}
\hline Variável & Média \pm desvio-padrão & Coeficiente de variação $(\%)$ \\
\hline AE $(\mathrm{cm})$ & $1,18 \pm 0,16$ & 13,6 \\
AO $(\mathrm{cm})$ & $0,86 \pm 0,08$ & 9,3 \\
VEd $(\mathrm{cm})$ & $1,65 \pm 0,23$ & 13,9 \\
VEs $(\mathrm{cm})$ & $0,93 \pm 0,20$ & 21,5 \\
SIVd $(\mathrm{cm})$ & $0,50 \pm 0,08$ & 16 \\
SIVs $(\mathrm{cm})$ & $0,73 \pm 0,10$ & 13,7 \\
PVEd $(\mathrm{cm})$ & $0,52 \pm 0,08$ & 15,4 \\
PVEs $(\mathrm{cm})$ & $0,71 \pm 0,11$ & 15,5 \\
AE:AO & $1,37 \pm 0,12$ & 8,8 \\
SIVd:PVEd & $0,96 \pm 0,17$ & 17,7 \\
SIVs:PVEs & $1,04 \pm 0,15$ & 14,4 \\
EPSS $(\mathrm{cm})$ & $0,09 \pm 0,04$ & 44,4 \\
$\% \Delta D(\%)$ & $43,83 \pm 8,39$ & 19,1 \\
ESS $(\%)$ & $47,17 \pm 15,34$ & 32,5 \\
ESP $(\%)$ & $36,38 \pm 14,97$ & 41,1 \\
FEj $(\%)$ & $77,34 \pm 9,11$ & 11,8 \\
Vcf $(\mathrm{cm} / \mathrm{s})$ & $2,42 \pm 0,56$ & 23,1 \\
TE $(\mathrm{s})$ & $0,18 \pm 0,02$ & 11,1 \\
\hline Varavel AE & ( $)$ & \\
\hline
\end{tabular}

Variável: $\mathrm{AE}$ = diâmetro do átrio esquerdo; $\mathrm{AO}$ = diâmetro da aorta; $\mathrm{VEd}=$ dimensão interna do ventrículo esquerdo na diástole; VEs = dimensão interna do ventrículo esquerdo na sístole; SIVd = espessura do septo interventricular na diástole; SIVs = espessura do septo interventricular na sístole; PVEd = espessura da parede posterior do ventrículo esquerdo na diástole; PVEs = espessura da parede posterior do ventrículo esquerdo na sístole; $\mathrm{AE}$ : $\mathrm{AO}=$ relação diâmetro átrio esquerdo e aorta; SIVd:PVEd = relação espessura do septo interventricular e da parede posterior do ventrículo esquerdo na diástole; SIVs:PVEs = relação espessura do septo interventricular e da parede posterior do ventrículo esquerdo na sístole; EPSS = distância entre o ponto $\mathrm{E}$ do folheto anterior da valva mitral e o septo interventricular; $\% \Delta \mathrm{D}=$ percentual de encurtamento sistólico do ventrículo esquerdo; \%ESS = percentual de espessamento sistólico do septo interventricular; \%ESP = percentual de espessamento sistólico da parede do ventrículo esquerdo; Fej = fração de ejeção; Vcf = velocidade de encurtamento circunferencial da fibra cardíaca; $\mathrm{TE}=$ tempo de ejeção. 
Observou-se correlação positiva e significativa com o peso corporal $(0,50 \leq r \leq 0,74$ e $0,001 \leq \mathrm{P} \leq 0,025), \quad$ a dimensão interna do ventrículo esquerdo na diástole (VEd) e na sístole (VEs), a espessura do septo interventricular na diástole (SIVd) e na sístole (SIVs), e a espessura da parede posterior do ventrículo esquerdo na diástole (PVEd) e na sístole (PVEs), assim como observado por Boon et al. (1983), Muzzi et al. (2000) e Yamato et al. (2006). Estes resultados eram esperados, uma vez que as dimensões cardíacas são proporcionais ao tamanho do animal. Entretanto, a distância entre o ponto $\mathrm{E}$ do folheto anterior da valva mitral e o septo interventricular (EPSS) não se correlacionou com o peso corporal, corroborando com Child et al. (1981). De acordo com esses autores, a EPSS independe do tamanho ventricular, apresentando alterações apenas quando a função encontra-se diminuída.

A idade do animal não se correlacionou com VEd, VEs, SIVs, EPSS, relação SIVd:PVEd e relação SIVs:PVEs $(-0,18 \leq \mathrm{r} \leq 0,34$ e $0,06 \leq \mathrm{P} \leq 0,43)$, mas observou-se baixa correlação $(0,43 \leq \mathrm{r} \leq 0,54$ e $0,001 \leq \mathrm{P} \leq 0,009) \quad$ com $\mathrm{SIVd}$, PVEd e PVEs. Estes resultados sugerem que, à medida que o animal envelhece, a PVE e o SIV tendem à hipertrofia, semelhante ao descrito por Boon et al. (1983).

Apenas a PVEd apresentou diferença significativa entre machos e fêmeas, semelhante ao observado por Crippa et al. (1992). As demais variáveis não apresentaram diferença entre os sexos. Essa não correlação era esperada, uma vez que havia homogeneidade de peso corporal entre machos e fêmeas.

A relação SIV:PVE é valiosa na avaliação dos padrões de hipertrofia - simétrica ou assimétrica. O valor encontrado neste estudo (Tab. 2) é semelhante aos de Boon et al. (1983), Muzzi et al. (2000) e Yamato et al. (2006). Marks (1993) mostrou que, na cardiomiopatia hipertrófica, a relação SIV:PVE é acima de 1,3.

A partir dos parâmetros ecocardiográficos, foram obtidos os índices de função ventricular esquerda, que são indicadores da contratilidade e complacência do ventrículo esquerdo. Assim como observado por Crippa et al. (1992), Muzzi (1998) e Yamato et al. (2006), os índices funcionais do $\mathrm{VE}$, quais sejam: percentual de encurtamento sistólico do ventrículo esquerdo
$(\% \Delta \mathrm{D})$, percentual de espessamento sistólico do septo interventricular (\%ESS) e da parede posterior do ventrículo esquerdo (\%ESP), e velocidade de encurtamento circunferencial da fibra cardíaca (Vcf), não apresentaram correlação significativa com peso corporal $(-0,16 \leq \mathrm{r} \leq-0,09 \mathrm{e}$ $0,21 \leq \mathrm{P} \leq 0,33)$. Da mesma forma, essas variáveis não se correlacionaram com idade ou sexo dos animais. A não correlação entre a idade e a maioria das variáveis estudadas era esperada, pois a faixa etária utilizada neste trabalho, entre um e seis anos, foi determinada com a finalidade de minimizar a influência de filhotes e animais idosos. Em relação ao sexo, Lombard (1984) e Morrison et al. (1992) também sugerem que esta variável não exerce influência sobre as medidas ecocardiográficas. Essas características são de grande importância clínica, pois possibilitam o uso de seus valores de referência em uma grande variedade de pacientes. Entretanto, alguns índices ecocardiográficos são influenciados pela frequência cardíaca, pré-carga e pós-carga, devendo ser avaliados com cautela (Boon, 1998).

As correlações entre peso corporal e idade e a significância de diferença entre sexos de todas as variáveis estudadas estão sumarizadas na Tab. 3.

O percentual de encurtamento sistólico do ventrículo esquerdo $(\% \Delta \mathrm{D})$ é um índice funcional, dependente da pré e pós-carga, amplamente utilizado na avaliação da função ventricular esquerda, devido à facilidade de cálculo e à alta correlação com as medidas invasivas (Boon et al., 1983). Boon et al. (1983) verificaram que a $\% \Delta \mathrm{D}$ independe da área de superfície corporal (ASC). Entretanto, outros autores concordam que pode existir variação dos valores normais da $\% \Delta \mathrm{D}$ entre raças de diferentes portes (Morrison et al., 1992; Koch et al., 1996; Silva et al., 2008). Geralmente, cães de raças grandes e gigantes apresentam valores mais baixos que os de raças pequenas. Snyder et al. (1995), Koch et al. (1996) e Muzzi et al. (2000), ao estudarem cães das raças Greyhound, Dogue Alemão e Pastor Alemão, respectivamente, encontraram valores médios de $28,8 \%, 25 \%$ e 28,6\%. Segundo Crippa et al. (1992), Yamato et al. (2006) e Silva et al. (2008), animais das raças Beagle, Poodle Miniatura e Schnauzer Miniatura, respectivamente, apresentam valores médios de $40 \%, 41,2 \%$ e $42,1 \%$, corroborando com o valor obtido no presente estudo, $43,8 \%$. 
Tabela 3. Correlação dos parâmetros ecocardiográficos com peso corporal e idade e significância de diferença entre sexos em cães da raça Yorkshire Terrier.

\begin{tabular}{cccc}
\hline Variável $^{(1)}$ & Peso corporal $^{(2)}$ & $\operatorname{Idade}^{(3)}$ & Sexo $^{(4)}$ \\
\hline AE $(\mathrm{cm})$ & 0,71 & $\mathrm{n}$ & - \\
AO $(\mathrm{cm})$ & 0,56 & $\mathrm{~ns}$ & - \\
VEd $(\mathrm{cm})$ & 0,74 & $\mathrm{~ns}$ & - \\
VEs $(\mathrm{cm})$ & 0,58 & $\mathrm{~ns}$ & - \\
SIVd $(\mathrm{cm})$ & 0,43 & - \\
SIVs $(\mathrm{cm})$ & 0,62 & $\mathrm{~ns}$ & - \\
PVEd $(\mathrm{cm})$ & 0,72 & 0,54 & 02 \\
PVEs $(\mathrm{cm})$ & 0,57 & 0,43 & - \\
AE;AO & 0,50 & $\mathrm{~ns}$ & - \\
SIVd:PVEd & $\mathrm{ns}$ & - \\
SIVs:PVEs & $\mathrm{ns}$ & $\mathrm{ns}$ & - \\
EPSS $(\mathrm{cm})$ & $\mathrm{ns}$ & - \\
$\% \Delta D(\%)$ & $\mathrm{ns}$ & $\mathrm{ns}$ & - \\
ESS $(\%)$ & $\mathrm{ns}$ & $\mathrm{ns}$ & - \\
ESP $(\%)$ & $\mathrm{ns}$ & $\mathrm{ns}$ & - \\
FEj $(\%)$ & $\mathrm{ns}$ & $\mathrm{ns}$ & - \\
Vcf $(\mathrm{cm} / \mathrm{s})$ & $\mathrm{ns}$ & $\mathrm{ns}$ & - \\
TE $(\mathrm{s})$ & $\mathrm{ns}$ & $\mathrm{ns}$ & - \\
\hline
\end{tabular}

(1) Variável: $\mathrm{AE}$ = diâmetro do átrio esquerdo; $\mathrm{AO}=$ diâmetro da aorta; $\mathrm{VEd}=$ dimensão interna do ventrículo esquerdo na diástole; VEs = dimensão interna do ventrículo esquerdo na sístole; SIVd = espessura do septo interventricular na diástole; SIVs = espessura do septo interventricular na sístole; PVEd = espessura da parede posterior do ventrículo esquerdo na diástole; PVEs = espessura da parede posterior do ventrículo esquerdo na sístole; $\mathrm{AE}: \mathrm{AO}=$ relação diâmetro átrio esquerdo e aorta; SIVd:PVEd = relação espessura do septo interventricular e da parede posterior do ventrículo esquerdo na diástole; SIVs:PVEs = relação espessura do septo interventricular e da parede posterior do ventrículo esquerdo na sístole; EPSS = distância entre o ponto E do folheto anterior da valva mitral e o septo interventricular; $\% \Delta \mathrm{D}=$ percentual de encurtamento sistólico do ventrículo esquerdo; \% ESS = percentual de espessamento sistólico do septo interventricular; \%ESP = percentual de espessamento sistólico da parede do ventrículo esquerdo; Fej = fração de ejeção; Vcf = velocidade de encurtamento circunferencial da fibra cardíaca; TE = tempo de ejeção.

${ }^{(2)(3)}$ Considerada significativa quando o coeficiente de correlação de Pearson $(r) \geq 0,40$ e $\mathrm{P}<0,05$. Se $\mathrm{r}<0,40$, a correlação foi considerada pouco ou não significativa (ns).

${ }^{(4)}$ Presente quando $\mathrm{P}<0,05$.

Tanto as medidas do septo interventricular (SIV) quanto da parede posterior do ventrículo esquerdo (PVE), na sístole e na diástole, foram utilizadas para calcular o percentual de espessamento sistólico do septo interventricular (\%ESS) e da parede posterior do ventrículo esquerdo (\%ESP). Essas duas variáveis indicam o grau de espessamento do SIV e da PVE, respectivamente, durante a contração ventricular, sendo também consideradas índices de função ventricular esquerda. Semelhantemente aos resultados de Boon et al. (1983), Muzzi et al. (2000) e Yamato et al. (2006), neste estudo não foi encontrada correlação significativa entre estas variáveis e peso corporal, sexo e idade (Tab. 3).

A fração de ejeção (FEj) é outro índice funcional muito utilizado na rotina ecocardiográfica, porém sua ampla faixa de variação torna seu uso limitado (Crippa et al., 1992). Cães de grande porte geralmente apresentam valores mais baixos que animais de raças pequenas (Snyder et al., 1995; Koch et al., 1996). O valor médio de FEj obtido neste trabalho, $77,3 \%$, foi semelhante aos encontrados por Crippa et al. (1992), Yamato et al. (2006) e Silva et al. (2008), que estudaram cães de pequeno porte das raças Beagle (77\%), Poodle Miniatura (74\%) e Schnauzer Miniatura $(75,1 \%)$, respectivamente.

A velocidade de encurtamento circunferencial da fibra cardíaca (Vcf) é a variação da circunferência da cavidade ventricular durante a sístole e incorpora na mesma medida o tempo de ejeção (TE) e a porcentagem de encurtamento sistólico do VE, apresentando boa aplicabilidade 
clínica. Entretanto, assim como a $\% \Delta \mathrm{D}$ e a FEj, sofre influência da pré e pós-carga. Neste estudo, o valor médio obtido, $2,42 \mathrm{~cm} / \mathrm{s}$, assemelha-se com aos encontrados por Boon et al. (1983) e Silva et al. (2008), de $2,07 \mathrm{~cm} / \mathrm{s}$ e $2,35 \mathrm{~cm} / \mathrm{s}$, respectivamente.

A distância entre o ponto $\mathrm{E}$ do folheto anterior da valva mitral e o septo interventricular (EPSS) também tem sido empregada na avaliação sistólica do VE. O EPSS não se altera com o tamanho da câmara ventricular, a não ser que a função esteja diminuída. Na presença de doenças valvulares ou de alterações na movimentação do septo interventricular, os valores de EPSS podem estar alterados, mesmo em cães com função normal (Koch et al. 1996; Kienle e Thomas, 2005). O resultado encontrado neste experimento, $0,9 \mathrm{~mm}$, condiz com a literatura, uma vez que a maioria dos relatos sugere que o valor de EPSS normal é mais baixo que $6 \mathrm{~mm}$.

\section{CONCLUSÕES}

A padronização dos valores ecocardiográficos para a raça Yorkshire Terrier é fundamental, pois auxilia no diagnóstico preciso de alterações cardíacas. Os parâmetros ecocardiográficos devem ser correlacionados com peso corporal, sexo e faixa etária, uma vez que são passíveis de variação, mesmo dentro de uma mesma raça canina.

\section{REFERÊNCIAS BIBLIOGRÁFICAS}

BOON, J.; WINGFIELD, W.E.; MILLER, C.H. Echocardiographic indices in the normal dog. Vet. Radiol., v.24, p.214-221, 1983.

BOON, J.A. Manual of veterinary echocardiography. Baltimore: Willians \& Wilkins, 1998. 478p.

CASTRO, M.G.; VEADO, J.C.C.; SILVA, E.F et. al. Estudo retrospectivo ecodopplercardiográfico das principais cardiopatias diagnosticadas em cães. Arq. Bras. Med. Vet. Zootec., v.61, p.1238-1241, 2009.

CAVALCANTI, G.A.O.; MUZZI, R.A.L.; ARAÚJO, R.B. et. al. Avaliação ecodopplercardiográfica da função diastólica em cães da raça Boxer. Arq. Bras. Med. Vet. Zootec., v.59, p.1169-1176, 2007.

CHILD, J. S.; KRIVOKAPICH, J.; PERLOFF, J. K. et al. Effect of left ventricular size on mitral $\mathrm{E}$ point to ventricular septal separation in assessment of cardiac performance. American Heart Journal, v. 101, n. 6, p. 797-805, 1981
CRIPPA, L.; FERRO, E.; MELLONI, P. et al. Echocardiographic parameters and indices in the normal Beagle dog. Lab. Anim., v.26, p.190-195, 1992.

FERIAN, P.E. Avaliação histológica, histoquímica, morfométrica, e radiográfica de traqueias de cães portadores de colapso traqueal. 2009. 100f. Tese (Doutorado em Medicina Veterinária) - Escola de Veterinária, Universidade Federal de Minas Gerais, Belo Horizonte, MG.

HANSSON, K.; HAGGSTROM, J.; KVART, C. et al. Left atrial root indices using two-dimensional and Mmode echocardiography in cavalier King Charles spaniels with or without left atrial enlargement. Vet. Radiol. Ultrasound, v.43, p.568-575, 2002.

KIENLE, R.D. Echocardiography. In: KITTLESON, M.D.; KIENLE, R.D. Small animal cardiovascular medicine. Baltimore: Mosby, 1998. p.95-117.

KIENLE, R. D.; THOMAS, W. P.Ecocardiografia. In: NYLAND, T.G.; MATTOON, J.S. Ultrassom diagnóstico em pequenos animais. 2.ed. São Paulo: Roca, 2005. p.365-438.

KOCH, J.; PEDERSEN, H.D.; JENSEN, A.L. et al. M-mode echocardiography diagnosis of dilated cardiomyopathy in giant breed dogs. J. Vet. Med., v.43, p.297-304, 1996.

LOMBARD, C.W. Normal values of the canine Mmode echocardiogram. Am. J. Vet. Res., v.45, p.20152018, 1984.

MARKS, C.A. Hypertrophic cardiomyopathy in a dog. J. Am. Vet. Med. Assoc., v.203, p.1020-1022, 1993.

MAROLF, A.; BLAIK, M.; SPECHT, A. A retrospective study of the relationship between tracheal collapse and bronchiectasis in dogs. Vet. Radiol. Ultrasound, v.48, p.199-203, 2007.

McREADY, D.M.; JOHNSON, L.R.; POLLARD, R.E. Fluoroscopic and radiographic evaluation of tracheal collapse in dogs; 62 cases $(2001-2006) . J$. Am. Vet. Med. Assoc., v.230, p.1870-1876, 2007.

MORRISON, S.A.; MOISE, N.S.; SCARLETT, J. et al. Effect of breed and body weight on echocardiographic values in four breeds of dogs of differing somatotypes. J. Vet. Int. Med., v.6, p.220224, 1992.

MUZZI, R.A.L. Ecodopplercardiografia em cães da raça Pastor alemão. 1998. 69f. Dissertação (Mestrado em Medicina Veterinária) - Escola de Veterinária, Universidade Federal de Minas Gerais, Belo Horizonte, MG. 
MUZZI, R.A.L.; ARAÚJO, R.B., MUZZI, L.A. L. et. al. Ecocardiografia modo $\mathrm{M}$ em cães normais da raça Pastor alemão (origem americana) do canil da Polícia Militar do Estado de Minas Gerais, Brasil. Cienc. Rural, v.30, p.819-824, 2000.

PAGE, A.; EDMUNDS, G.; ATWELL, R.B Echocardiographic values in the Greyhound. Aust.Vet. J., v.70, p.361-364, 1993.

PELLEGRINO, A.; PETRUS, L.C.; PEREIRA, G.G. et. al. Padronização de parâmetros ecocardiográficos de cães da raça Golden Retriever clinicamente sadios. Cien. Rural, v.37, p.1039-1044, 2007.

RISHNIW, M.; ERB, H.N. Evaluation of four 2dimensional echocardiographic methods of assessing left atrial size in dogs. J. Vet. Int. Med., v.14, p.429435, 2000.

SAMPAIO, I.B.M. Estatística aplicada à experimentação animal. 2.ed. Belo Horizonte: FEPMVZ, 2002. 265p.
SILVA, E.F.; MELO, M.B.; MUZZI, R.A.L. et al. Índices ecodopplercardiográficos de função ventricular esquerda em cães das raças Boxer e Schnauzer miniatura. Arq. Bras. Med. Vet. Zootec., v.60, p.71-75, 2008.

SNYDER, P.S.; SATO, T.; ATKINS, C.E. A comparison of echocardiographic indices of the nonracing, healthy Greyhound to reference values from other breeds. Vet. Radiol. Ultrasound, v.36, p.387-392, 1995.

THOMAS W.P.; GABER, C.E.; JACOBS, G.J. et al. Recommendations for standards in transthoracic twodimensional echocardiography in the dog and cat. $J$. Vet. Int. Med., v.7, p.247-252, 1993.

YAMATO, R.J.; LARSSON, M.H.M.A.; MIRANDOLA, R.M.S. et al. Parâmetros ecocardiográficos em modo unidimensional de cães da raça Poodle miniatura, clinicamente sadios. Cienc. Rural, v.36, p.142-148, 2006. 